\title{
Reaction of Congo Red in Water after Irradiation by Pulsed Intense Relativistic Electron Beam
}

\author{
Takashi KIKUCHI, Hiroshi MORIWAKI ${ }^{1)}$, Hiromitsu NAKANISHI ${ }^{2)}$, Hironobu KONDO, \\ Toru SASAKI, Go IMADA ${ }^{3,4)}$ and Nob. HARADA \\ Department of Electrical Engineering, Nagaoka University of Technology, Niigata 940-2188, Japan \\ ${ }^{1)}$ Division of Applied Biology, Faculty of Textile Science and Technology, Shinshu University, Nagano 386-8567, Japan \\ ${ }^{2)}$ Satellite Venture Business Laboratory, Shinshu University, Nagano 386-8567, Japan \\ ${ }^{3)}$ Department of Information and Electronics Engineering, Niigata Institute of Technology, Niigata 945-1195, Japan \\ ${ }^{4)}$ Extreme Energy-Density Research Institute, Nagaoka University of Technology, Niigata 940-2188, Japan
}

(Received 14 February 2011 / Accepted 11 April 2011)

\begin{abstract}
The reaction of congo red, a well-known toxic azo dye, occurred after irradiation by a pulsed intense relativistic electron beam (PIREB). An aquation of congo red was irradiated by PIREB (2 MeV, 0.36 kA, $140 \mathrm{~ns}$ ). After PIREB irradiation, the solution was measured by electrospray ionization-mass spectrometry and liquid chromatography/mass spectrometry. It was found that congo red underwent a reaction ( $77 \%$ conversion after five shots of PIREB irradiation) and the hydroxylated compounds of the dye were observed as reaction products.
\end{abstract}

(C) 2011 The Japan Society of Plasma Science and Nuclear Fusion Research

Keywords: pulsed intense relativistic electron beam (PIREB), azo dye, congo red

DOI: $10.1585 /$ pfr.6.1206021

The safety of water supplies and aquatic conservation are pressing problems. Therefore, it is important to develop technologies for the decomposition of the pollutants in water; various water cleanup techniques have received much attention [1].

A pulsed intense relativistic electron beam (PIREB) has been applied as a technology for environmental cleanup. For example, multi-shot PIREB irradiation reportedly decreased the NOx concentration in a chamber, inactivated the zooplankton in water, and treated the volatile organic compounds contained in soil [2]. PIREBs have great potential for various treatment effects because they not only provide electrons with high kinetic energy but also generate radicals and X-ray emission. In comparison with the treatment method using a DC electron beam [3], the PIREB method has the advantage of offering brief treatments because the beam current is on the order of $\mathrm{kA}$ with a short pulse duration.

In this study, we demonstrated the degradation of congo red, a well-known toxic azo dye, by the PIREB treatment. Azo dyes are widely used in various products and leak out with industrial wastewater into the environment. Therefore, the development of a treatment technique for the removal or decomposition of azo dyes is very important.

Figure 1 shows the experimental setup. The electrons emitted from the cathode are accelerated by the applied voltage in the diode gap. The ring anode generates an electron beam with a hollow shape. Because the applied volt-

author'se-mail: tkikuchi@vos.nagaokaut.ac.jp

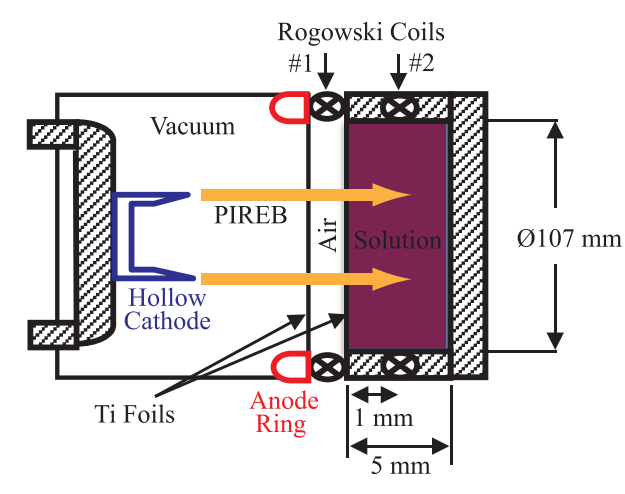

Fig. 1 Experimental setup of PIREB irradiation of a reactor containing a congo red solution.

age is $2 \mathrm{MV}$ in the diode gap in a vacuum (0.02 Pa), PIREB can be extracted to the outside of the diode. PIREB travels through air space after passing through a Ti foil and irradiates $45 \mathrm{~mL}$ of a congo red aquation $(0.10 \mathrm{mM})$ in a reactor containing Ti foil. The reactor was spatially isolated from the diode. To generate PIREB, we used the first acceleration cell of the pulsed power generator ETIGO-III [4], which is an electron induction accelerator.

Figures 2 and 3 show the typical voltage and beam current waveforms at the electron-beam diode and Rogowski coils, respectively. As shown in Fig. 2, the voltage and current corresponding to the extraction voltage and the beam current of PIREB reached $-2 \mathrm{MV}$ and $-4 \mathrm{kA}$ at the diode, respectively. As shown in Fig. 3, PIREB with a peak current of $-0.36 \mathrm{kA}$ entered the solution in the reactor. 
Figure 4 shows the congo red solutions before and after PIREB irradiation. After PIREB irradiation, the congo red solution changed color from red to dark red. The color of a congo red aquation is well known to deepen with decreasing $\mathrm{pH}$. Therefore, the color change is believed to

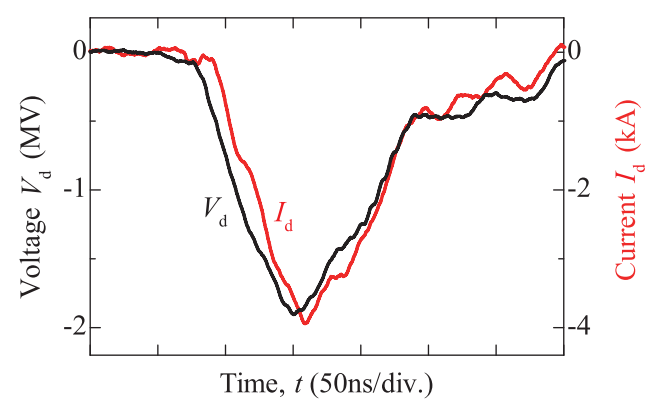

Fig. 2 Typical waveforms of voltage and current at the diode.

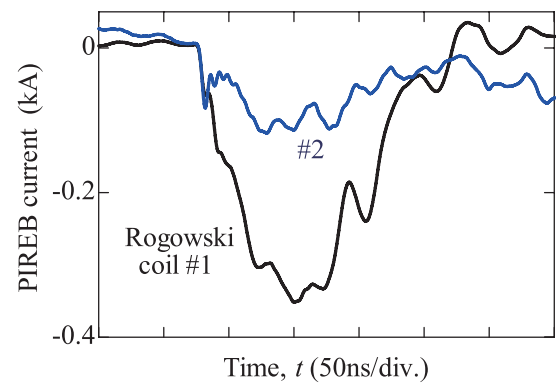

Fig. 3 Typical waveforms of PIREB current detected at each Rogowski coil as shown in Fig. 1. result from change in $\mathrm{pH}$.

After irradiation, the solution was analyzed by electrospray ionization-mass spectrometry (ESI/MS) and liquid chromatography/mass spectrometry (LC/MS) in order to identify the reaction products and to quantify the amount of congo red, respectively. An LC/MS 2010A mass spectrometer (Shimadzu, Kyoto, Japan) was used for the ESI/MS measurement (negative ion mode). The ESI/MS conditions were as follows: scan range, $m / z$ 100-800; heat block temperature, $200^{\circ} \mathrm{C}$; interface voltage, $4.5 \mathrm{kV}$; CDL voltage, $20 \mathrm{~V}$. The solutions $(10 \mu \mathrm{L})$ were injected into the LC/MS system; the flow rate of the mobile phase (acetonitrile) was $0.20 \mathrm{~mL} / \mathrm{min}$. In addition, the LC/MS 2010A instrument was used to quantify the amount of congo red. Amide- 80 (Tosoh, Tokyo, Japan: $2.0 \times 150 \mathrm{~mm}$ i.d.) was used for LC separation. High-performance LC separation was performed at $40^{\circ} \mathrm{C}$ using a gradient composed of solution A (6.5 mM ammonium acetate solution adjusted to $\mathrm{pH} 5.5$ ) and solvent B (acetonitrile). The gradient conditions were as follows: $0-5 \mathrm{~min}, 100 \%$ solvent $\mathrm{B} ; 5-12 \mathrm{~min}$, a linear decrease from 100 to $25 \% \mathrm{~B} ; 12-15 \mathrm{~min}$, hold at $25 \% \mathrm{~B}$. The flow rate was $0.20 \mathrm{~mL} / \mathrm{min}$. The ESI conditions were

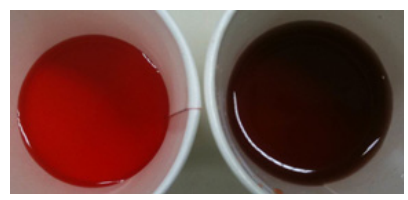

Fig. 4 Photographs of congo red solutions before (left) and after (right) PIREB irradiation.

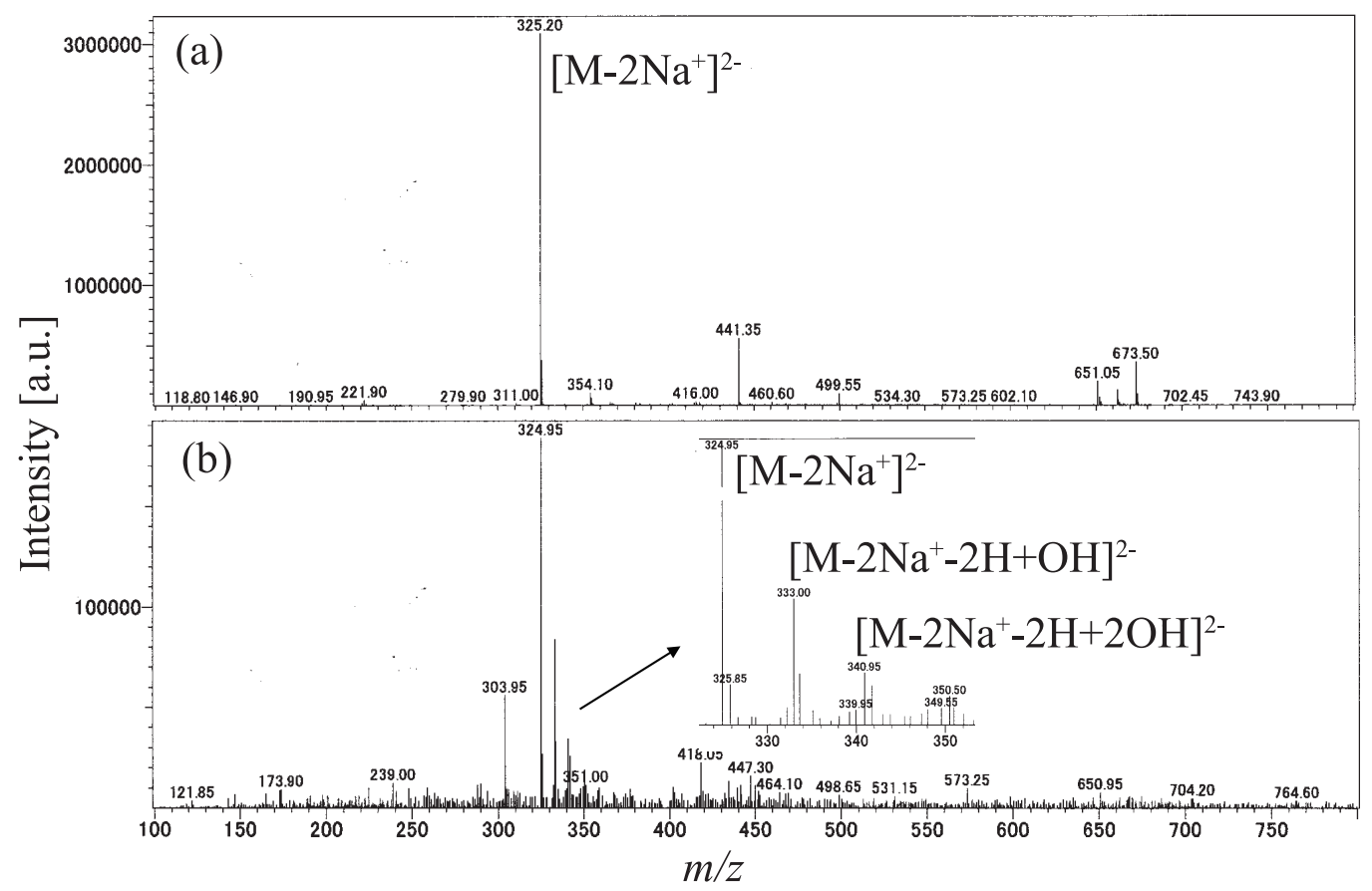

Fig. 5 Mass spectrum of congo red solution before (a) and after (b) PIREB irradiation (five shots). 
the same as those described above. The reaction solutions were diluted 100 times and injected into the LC/MS system. LC/MS acquisition was performed in the selected ion monitoring mode at $m / z 325$.

Figure 5 shows the ESI mass spectra of the solutions before and after PIREB irradiation (five shots). The ion peaks assignable to the hydroxylated compounds of congo red (the divalent ion of the monosubstituted compound, $m / z$ 333; the divalent ion of the disubstituted compound, $m / z$ 340) were observed by ESI/MS. The ion peaks corresponding to the products were not observed by ESI/MS in the positive ion mode. The hydroxylated compounds of congo red have reportedly been formed by $\gamma$-ray irradiation [5]. Radiation-induced oxidative or reductive species such as $\mathrm{OH}$ radicals or aqueous electrons $\mathrm{e}_{a q}^{-}$degraded congo red in the aqueous solution. It is also well-known that hydroxyl radicals are formed by the radiolysis of water [6]. Therefore, a similar reaction is believed to have occurred in the PIREB reaction. In addition, the absorption spectra of the congo red solution were measured before and after PIREB irradiation after adjusting the $\mathrm{pH}$ value to 6 (see Fig. 6). The adjustment of $\mathrm{pH}$ was required because the color of the congo red solution changes with $\mathrm{pH}$ (Fig. 4). The peak at $500 \mathrm{~nm}$ was decreased by PIREB irradiation. This result indicates that the $\pi$-electron conjugated system of congo red was broken by the attack of the reaction species formed by PIREB irradiation on the azo group of congo red. That is, it is possible that the products, which consist of the broken azo group and cannot be detected by ESI/MS, resulted from PIREB irradiation. The LC/MS results indicated congo red conversion rates of $45 \%$ after one shot of PIREB irradiation and $77 \%$ after five shots.

A combined electron beam and biological treatment was previously used to purify dyeing wastewater [3]. In this report, we found that congo red was reacted by PIREB irradiation. PIREB irradiation is expected to be applied to

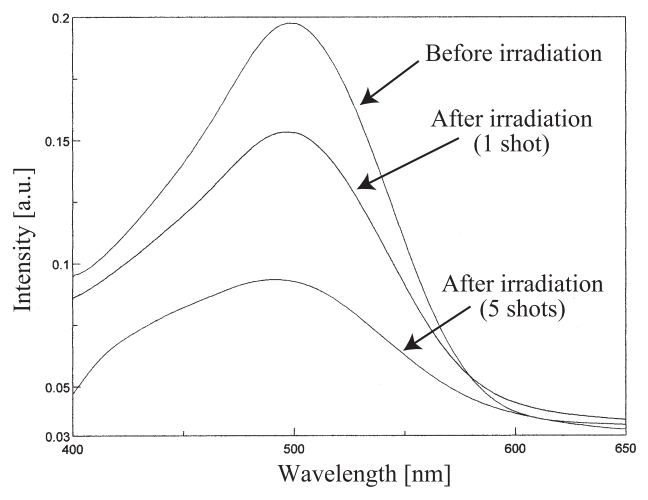

Fig. 6 Changes in the UV spectra of congo red aquation after PIREB irradiation.

the decomposition of azo dyes other than congo red and other environmental pollutants in water. However, there is no evidence that PIREB irradiation decreased the toxicity of the congo red solution. It is necessary to explore any change in the toxicity of the solution after PIREB irradiation in order to apply the technique to the decomposition of pollutants in water. In addition, downsizing of the PIREB irradiation equipment and reducing the initial cost of the equipment are important problems for the widespread application of PIREB as an environmental technology.

[1] H. Moriwaki et al., J. Hazard. Mater. 185, 725 (2011).

[2] G. Imada and K. Yatsui, IEEE Trans. Plasma Sci. 31, 295 (2003); H. Kondo et al., Plasma Fusion Res. 5, 036 (2010); G. Imada, IEEJ Trans. 6, 88 (2011).

[3] B. Han et al., Radiat. Phys. Chem. 64, 53 (2002).

[4] A. Tokuchi et al., Proc. 12th Int'l Conf. High Power Particle Beams (1998) p.175.

[5] H. Ma et al., Chemosphere 68, 1098 (2007).

[6] C.D. Jonah and J.R. Miller, J. Phys. Chem. 81, 1974 (1977). 